Regular Paper

\title{
A Coil for Wireless Power Transmission by Using Sewing Technique with Litz Wire
}

\author{
Ryohei SUWAHARA (Stu. Mem.), Ryota KATO (Stu. Mem.), Koki MURATA (Stu. Mem.), \\ Tomoki WATANABE (Stu. Mem.), Yuichi SHIMATANI and Shogo KIRYU (Mem.)
}

\begin{abstract}
A Coil for wireless power transmission was manufactured by using of sewing techniques. The sewing methods and electric characteristics were described. The coils were sewn with a cross stitch. The inductance and loss resistance of the sewn coils was measured. It is assumed that the power receiving side coil is attached to biomedical implantable devices. The coupling coefficient between the power receiving side coil and the sewn coil was measured. The maximum coupling coefficient was 0.75 . The maximum efficiency of $75.0 \%$ was obtained when the coupling coefficient was maximum. Inverter and rectifier were attached the circuit and the load voltage was measured. The maximum load voltage was $4.77 \mathrm{~V}$.
\end{abstract}

Keywords: wireless power transmission, sewn coil, magnetic resonance

(Received: 22 July 2018, Revised: 19 December 2018)

\section{Introduction}

In recent years, smart textiles have been drawing attention as a new wearable device following smart watches and smart glasses [1]. Some devices in the smart textiles can be fabricated on clothes. They, therefore, can be set as close to the human body as possible. Due to its characteristic, it is considered to be applied to devices for measuring a human vital sensor such as blood pressure, heart rate, and calorie consumption [2]. Based on the idea of these smart textiles, there was an example in which an inductor was made in clothing and made into an antenna [3]. Power transmission with the technologies was attempted [4], though the application was not clear. A coil fabricated on the clothes can be used in the wireless power transmission to biomedical implantable devices such as cardiac pacemakers, Implantable Cardioverter Defibrillators (ICD), cochlear implants, and so on. Wireless power transmission technology to bio implantation devices is studied in the transcutaneous energy transmission system (TETS) to the artificial ancillary heart. Some of these technologies have been put to practical use [5]. Fig. 1 shows a schematic diagram of power transfer to an implant device using a coil sewn on clothes.

Litz wire is often used to fabricate coils with low conductive loss and skin effect at a higher frequency. The wire is also flexible enough to be sewn to fabric. Therefore, spiral coils can be directly manufactured to fabric such as clothes by using the wire. A paper comparing the coil made by hand stitched and the coil

\footnotetext{
Correspondence: Ryohei Suwahara, Biomedical Engineering, Tokyo City University, 1-28-1 Tamazutsumi, Setagaya-ku, Tokyo 158-8557, Japan email: g1781413@tcu.ac.jp

${ }^{* 1}$ Tokyo City University
}

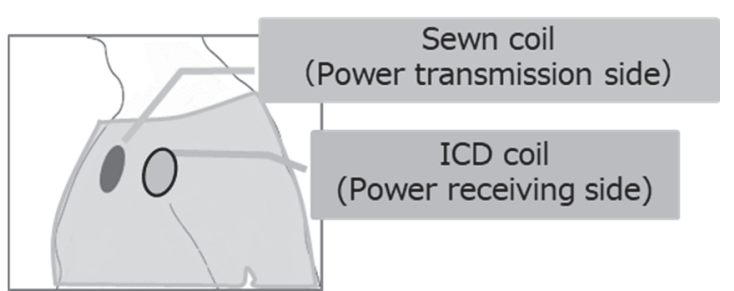

Fig. 1 Expected transmission to the implantable device

of Qi standards was published in Japan [6]. In addition, there is case in which the coils for power transmission are sewn into various fabrics using a sewing machine [7].

The flexibility and breathability of the part where the coil is sewn can be adjusted by changing the pitch of the coil. These coils can be more convenient for the above applications than conventional coils because the power can be supplied to the implantable devices from the clothes with the sewn coil. As an example, a coil sewn into a glove made of knitted cloth was shown in Fig. 2. The coil is used for power supply to a smart phone held in the hand. The power source used a mobile battery. The battery was placed in a small pocket on the back of the glove.

In this paper, the manufacturing methods of the coils sewn with Litz wire were described. The coupling coefficients and the transmission efficiencies were measured between the sewn coil and a receiver coil which was designed to set in an ICD. The magnetic field resonance type was adopted as the transmission method. The frequency range of those experiments was $100 \mathrm{kHz}$ used in the Qi standard. In practical use, a DC battery will be used as the power supply for the transmitter circuit, and a DC voltage will be required to charge the ICD device. An inverter in the transmitter side and a rectifier in the receiver side will be necessary. The DC load voltage of $4.0 \mathrm{~V}$ or higher is necessary to charge the lithium ion battery. In order to show the practicality of 


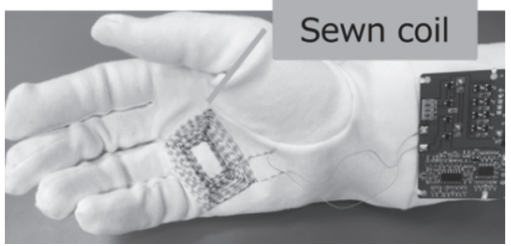

Fig. 2 Glove with stitching coil

the sewn coil, a wireless power transmission circuit was fabricated with a inverter and a rectifier.

\section{Manufacturing Method}

In this section, the method to manufacture the sewn coil with Litz wire is described. The Litz wire used in the fabrication has a diameter $0.6 \mathrm{~mm}(0.04 \mathrm{~mm} \times 100)$. Hand stitch was used in the production. The needle used in the sewing was a tapestry needle No.20. The fabric (sometimes called Java canvas) used in the coil production was 11 count aida cloth. Aida cloth is an open weave cotton fabric. The fabric is composed of blocks with the several warp and woof threads. The sewn coil was assumed to be used to supply electric power to an ICD. The typical size of ICDs is about $70 \mathrm{~mm}$ in length, about $50 \mathrm{~mm}$ in width and about $10 \mathrm{~mm}$ in thickness. In order to set the receiver coil in the ICD, the shape of that was determined to be a circular spiral shape with an outer diameter of $40 \mathrm{~mm}$. The transmission coil was assumed to supply power to the ICD under the skin. The thickness of the skin is said to be $1 \mathrm{~mm}$ to $2 \mathrm{~mm}$ [8]. High transmission efficiency can be obtained as the coupling coefficient between the coils is larger. Therefore, the diameter of the sewn coil which can obtain the highest coupling coefficient was obtained by using the finite element method. The diameter of the sewn coil was varied from $40 \mathrm{~mm}$ to $60 \mathrm{~mm}$. The vertical distance between the opposing coils was set at $5 \mathrm{~mm}$. One coil was moved in the horizontal direction, and the coupling coefficient was simulated. The simulation results are shown in Fig 3.

The maximum coupling coefficient 0.59 was obtained when the diameter of the sewing coil was $50 \mathrm{~mm}$ and 55 $\mathrm{mm}$. The reduction factor of the coupling coefficient was smaller for coils with a diameter of $55 \mathrm{~mm}$ than for coils with a diameter of $50 \mathrm{~mm}$. From those things, the diameter of the sewn coil was determined to be $55 \mathrm{~mm}$.

The fabrication process of the sewn transmitter coil is as follows. First, the center of the coil and the outer diameter were marked. Second, the Litz wire was sewn around the point to be in the shape of a circle. The wire was sewn from the inside to the outside. Finally, after sewing the Litz wire, the loosely fabricated coil was firmly fixed with conventional thread by hand stitch in the radial direction. The manufacturing process of the coil is shown in Fig.4.

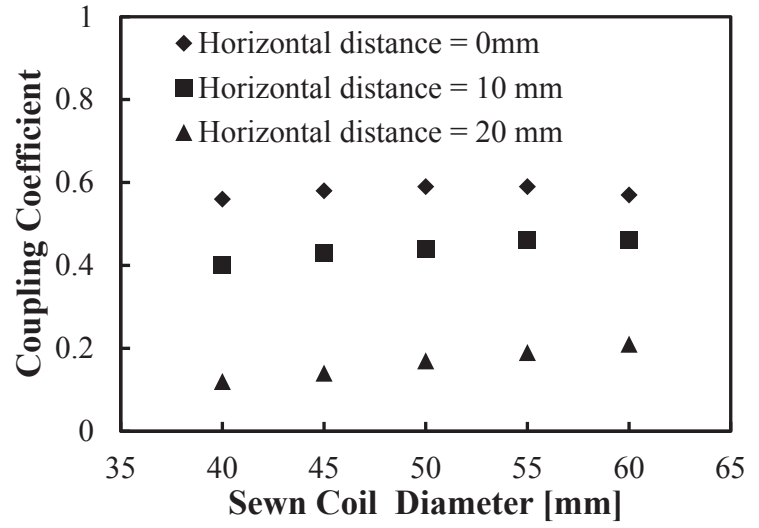

Fig. 3 The sewn coil diameter dependence of coupling coefficient.

1

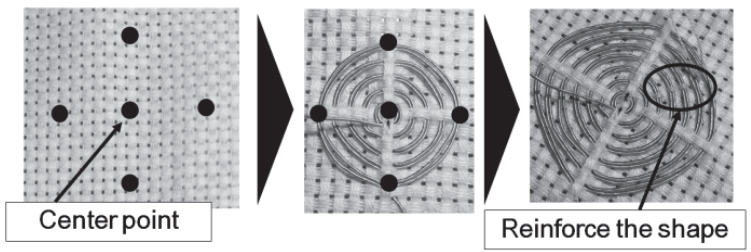

Fig. 4 Production process of sewn coil.

In the hand stitch, kinks of the wire are carefully avoided especially when a large number of turn is needed. The occurrence of kink in the coil manufacturing process may cause the coating to peel off.

\section{Electrical Characteristics}

The coils used for wireless power transmission is shown in Fig. 5. In Table 1 show the number of turns, inner diameter and outer diameter. The inductance and loss resistance of the coil were measured with a frequency of $100 \mathrm{kHz}$ to $5500 \mathrm{kHz}$ using an LCR meter. The measurable maximum frequency of the LCR meter used was $5500 \mathrm{kHz}$. The frequency characteristic of the inductance is shown in Fig. 6. Fig. 7 shows the frequency characteristic of the loss resistance. The inductances and resistances of the coils were increased with the frequency. The measured resistance was composed of direct current resistance of copper wire, resistance by the skin effect, and resistance by the proximity effect [9-11]. Since the diameter of the line constituting the Litz wire was sufficiently small, influence of the skin effect is not seen in the sewn coil.

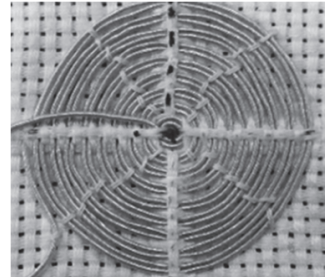

(a) Sewn coil

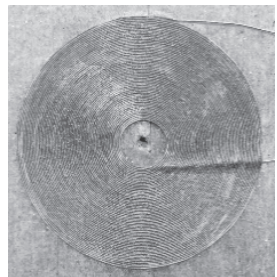

(b) Receiver coil
Fig. 5 The coils used for wireless power transmission. 
Table 1 Number of Turns and Size of the Coils.

\begin{tabular}{|c|c|c|}
\hline & Sewn coil & Receiver coil \\
\hline Turns & 26 & 57 \\
\hline Inner Dia. $(\mathrm{mm})$ & 1 & 3.8 \\
\hline Outer Dia. $(\mathrm{mm})$ & 55 & 40 \\
\hline
\end{tabular}

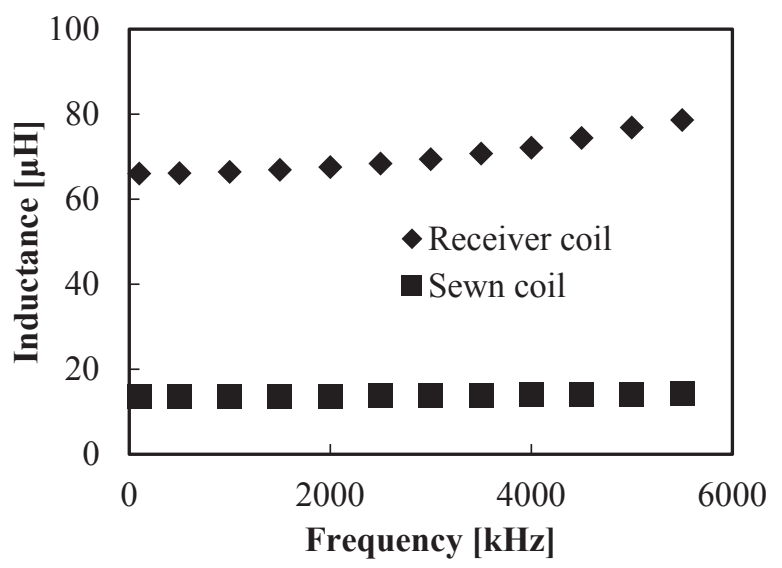

Fig. 6 Frequency characteristics of the inductance.

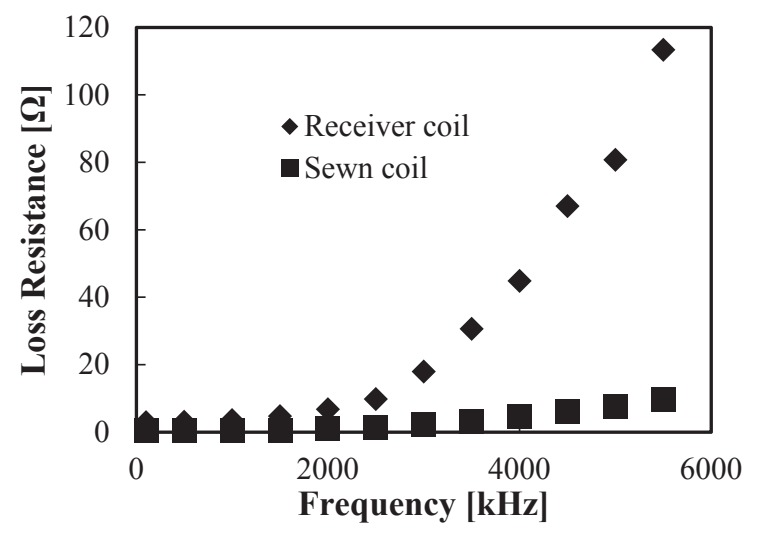

Fig. 7 Frequency characteristics of the loss resistance.

\section{Coupling Coefficients between Sewn Coil and Receiver Coil}

The coupling coefficients between the sewn coil and the receiver coil were calculated from the self and the mutual inductance measured by an LCR meter at a frequency of $100 \mathrm{kHz}$. The inductances were measured in distances between different coils. First, the centres of the two coils were aligned. Second, the coils were closed contact with each other. The position of these coils was determined as a reference. Furthermore, the coupling coefficient was measured by moving one coil vertically and horizontally. Changes in distance between coils in the vertical direction were $0 \mathrm{~mm}, 5 \mathrm{~mm}$, and $10 \mathrm{~mm}$. Changes in the horizontal direction were $0 \mathrm{~mm}, 5 \mathrm{~mm}$, $10 \mathrm{~mm}, 15 \mathrm{~mm}$, and $20 \mathrm{~mm}$. Fig. 8 shows the coil position in the measurement of the coupling coefficient.

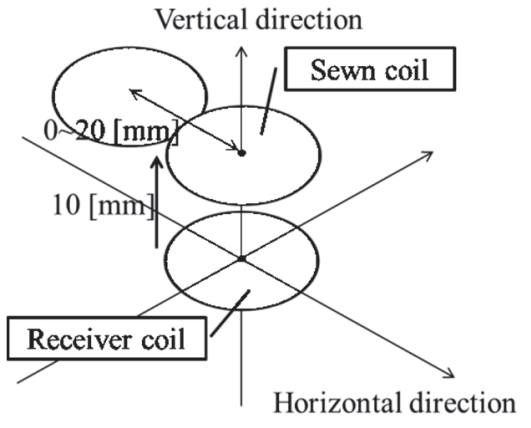

Fig. 8 Coil position in measurement of coupling coefficient.

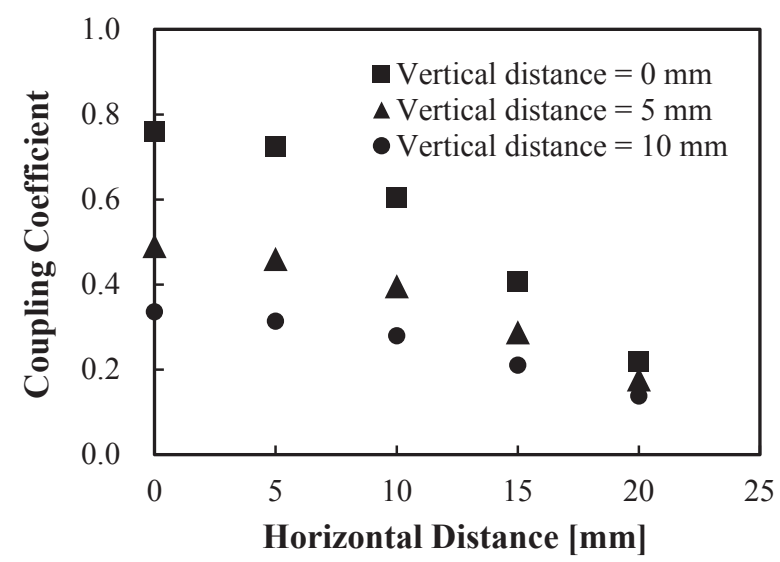

Fig. 9 Distance dependence of the coupling coefficient.

Fig. 9 show the measurement results in each situation. In Fig. 9, the maximum coupling coefficient 0.76 was obtained when the coils were set to the closest position. The measured minimum coupling coefficient was 0.14 when the coils were set to the furthest position. The coupling coefficient was gently decreased when one coil was moved in the horizontal direction.

\section{Transmitting Efficiency}

\subsection{Experimental Method}

Fig. 10 shows an equivalent circuit used for measuring the power transmission efficiency. The positions of the sewn coil and the receiver coil are the same as in Fig. 8. The frequency used for the measurement was set to $100 \mathrm{kHz}$ with reference to the Qi standard. The inductance of the sewn coil was indicated with $L_{1} . L_{2}$ was that of the receiver coil. The coupling coefficient between them was indicated with $k . V$ is the voltage of the power source with the internal resistance $R_{0}$. The conduction loss of the coils is represented to be $R_{L 1}$ for the transmitter side and $R_{L 2}$ for the receiver. The load resistance is shown by $R_{L}$. $R_{S}$ is a resistance by which the transmitter current is measured. Resonant capacitors are shown by $C_{1}$ and $C_{2} . R_{C l}$ and $R_{C 2}$ are the equivalent series resistance of the capacitor. 


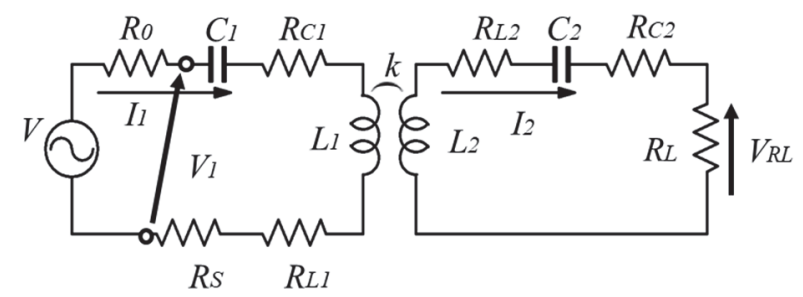

Fig. 10 An equivalent circuit of an electromagnetic induction wireless power transfer system.

Table 2 Experimental Circuit Components and Value.

\begin{tabular}{|c|c|c|}
\hline Components & Part & Value \\
\hline Power Supply Voltage & $V$ & $5.0(\mathrm{~V})$ \\
\hline $\begin{array}{c}\text { Inductance of Transmitter } \\
\text { Coil }\end{array}$ & $L_{1}$ & $13.6(\mu \mathrm{H})$ \\
\hline Inductance of Receiver Coil & $L_{2}$ & $66.0(\mu \mathrm{H})$ \\
\hline $\begin{array}{c}\text { Power Supply Internal } \\
\text { Resistance }\end{array}$ & $R_{0}$ & $0.5(\Omega)$ \\
\hline $\begin{array}{c}\text { Conduction Loss of the } \\
\text { Transmitter Coil }\end{array}$ & $R_{L 1}$ & $0.38(\Omega)$ \\
\hline $\begin{array}{c}\text { Conduction Loss of the } \\
\text { Receiver Coil }\end{array}$ & $R_{L 2}$ & $2.99(\Omega)$ \\
\hline Load Resistance & $R_{L}$ & $39.6(\Omega)$ \\
\hline $\begin{array}{c}\text { Resistance for Current } \\
\text { Detection }\end{array}$ & $R_{S}$ & $0.5(\Omega)$ \\
\hline $\begin{array}{c}\text { Transmitter side resonance } \\
\text { capacitor }\end{array}$ & $C_{I}$ & $0.18(\mu \mathrm{F})$ \\
\hline $\begin{array}{c}\text { Receiver side resonance } \\
\text { capacitor }\end{array}$ & $C_{2}$ & $0.04(\mu \mathrm{F})$ \\
\hline $\begin{array}{c}\text { Equivalent series resistance } \\
\text { of transmitter side capacitor }\end{array}$ & $R_{C l}$ & $0.1(\Omega)$ \\
\hline $\begin{array}{c}\text { Equivalent series resistance } \\
\text { of receiver side capacitor }\end{array}$ & $R_{C 2}$ & $0.6(\Omega)$ \\
\hline
\end{tabular}

The current $I_{1}$ flows in the transmitter side and $I_{2}$ in the receiver side. Table 2 shows circuit components and the values. Based on Fig. 10, the power transmission efficiency $\eta$ is given as (1):

$$
\eta=\frac{\frac{V_{R L}{ }^{2}}{R_{L}}}{V_{1} I_{1} \cos \theta}
$$

In Equation 1, $V_{R L}$ is the voltage applied to the load resistance. $V_{l}$ is a voltage applied to the power transmission side circuit excluding the power supply resistor $R_{0} . \theta$ is the phase difference of the power transmission side circuit. The theoretical equation of the transmission efficiency in the magnetic field resonance type is given by the equation 2 :

$$
\eta^{\prime}=\frac{\omega^{2} k^{2} L_{1} L_{2} R_{L}\left(R_{1}+R_{2}\right)}{R_{2}\left(R_{1}+R_{2}-R_{0}\right)\left(R_{1} R_{2}+\omega^{2} k^{2} L_{1} L_{2}\right)}
$$

In Equation 2, the combined resistance on the transmission side was indicated by $R_{1}$. The combined resistance on the power receiver side is indicated by $R_{2}$.

\subsection{Measurement Result}

Fig. 11 (a), (b) and (c) show the transmission efficiency measured at each vertical distance. The transmission efficiency of each coupling coefficient is shown in Fig. 12. In Fig. 11 (a), (b) and (c) the highest power efficiency was obtained at the position where the coupling coefficient of the two coils was the highest. The maximum transmission efficiency was $75.0 \%$ in Fig. 11 (a). In Fig. 11 (b), the maximum transmission efficiency was $56.0 \%$. The maximum transmission efficiency was $35.9 \%$ in Fig. 11 (c). The maximum difference between the measured value and the theoretical value was $8.3 \%$. One of the reasons for the difference is caused by the measurement uncertainty. And the other is ignoring the inductance of the wirers of the circuit in theoretical calculation.

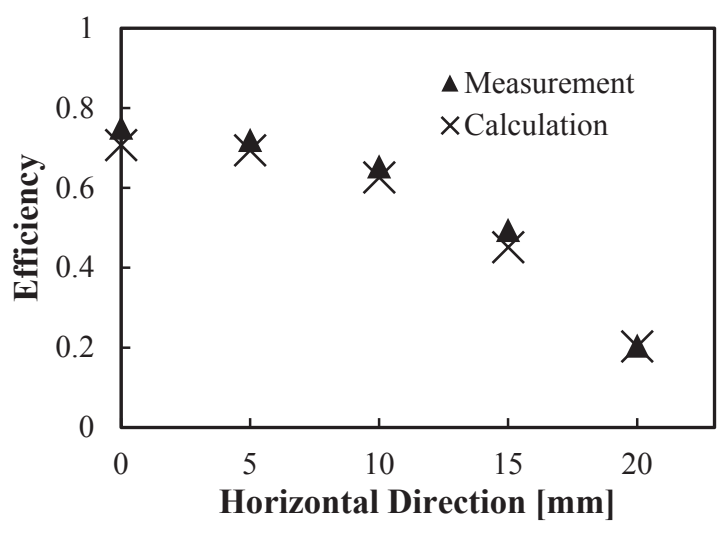

(a) Vertical Distance $=0 \mathrm{~mm}$

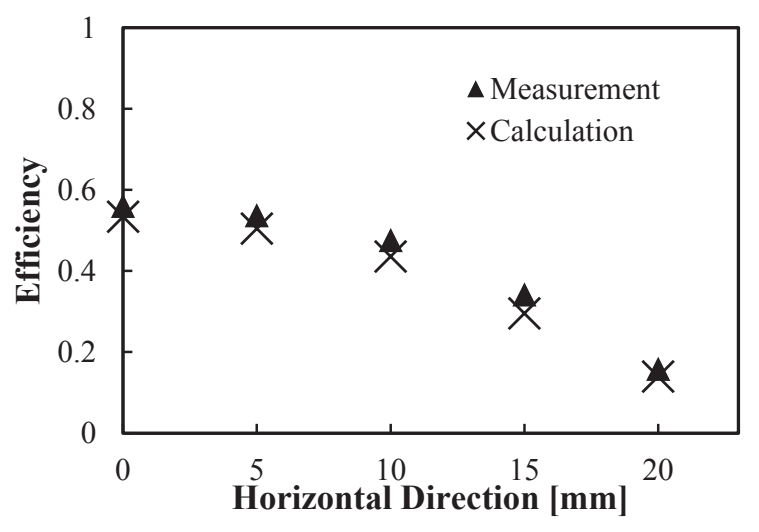

(b) Vertical Distance $=5 \mathrm{~mm}$ 


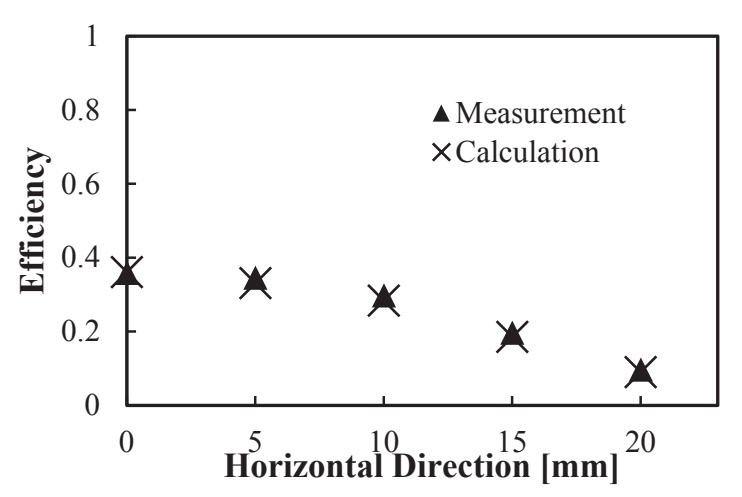

(c) Vertical Distance $=10 \mathrm{~mm}$

Fig. 11 Transmission efficiency at resonance point.

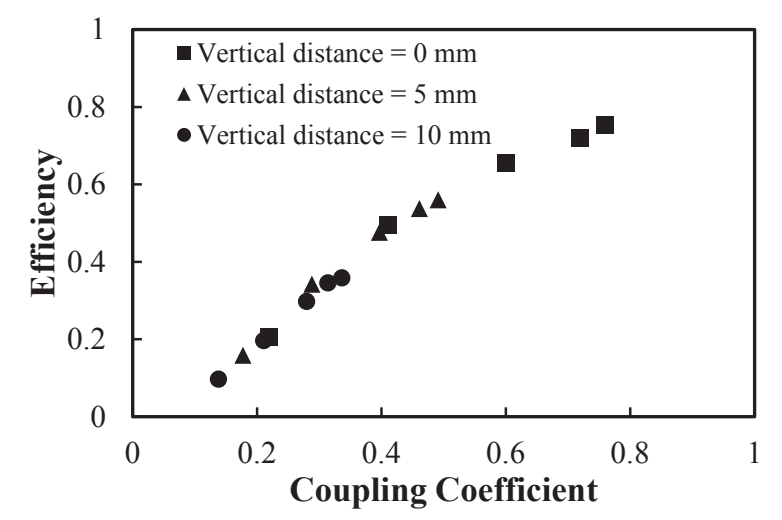

Fig. 12 Transmission efficiency for each coupling coefficient.

\section{DC Load Voltage Measurement}

The charging method of the secondary battery is the CC-CV (Constant Current - Constant Voltage) method. The method charges with a $\mathrm{CC}$ until it reaches a specific voltage, and changes to charging with a $\mathrm{CV}$ when reaching the set voltage. The load voltage of $4.0 \mathrm{~V}$ or higher is required, when charging this method. Based on that voltage, a practical coupling coefficient range for wireless power transmission using the sewn coil was investigated. The load voltage was measured by providing an inverter and a rectifier.

The inverter was a half bridge to avoid increasing the number of elements of the circuit. The Qi standard power transmitter design referred to A10 [12]. The circuit used for the measurement is shown in Fig. 13. The inductance of the sewn coil was indicated with $L_{1}$. $L_{2}$ was that of the receiver coil. The coupling coefficient between them was indicated with $k . V_{i n}$ is the DC voltage of the power source. $R_{\mathrm{S}}$ is the resistor to measure the output current $I_{i n}$. The load resistance is shown by $R_{\mathrm{L}}$. The FET constituting the inverter was indicated $S_{1}$ and $S_{2} . C_{3}$ and $C_{4}$ show shunt capacitors. The smoothing capacitor is $C_{5} . R_{C}$ indicate a Charge release resistance. The schottky diode of the rectifier was indicated by $D$. Table 3 shows circuit components and the values.

The circuit on the power transmission side is the class DE inverter. In the circuit, a shunt capacitor was inserted in parallel with the MOSFET of the class D inverter. The capacitors create time for both switches to turn off and optimize the switching conditions [13]. It is expected that the switching loss decrease and the load voltage increase. The switching element of the circuit used in the experiment was the low voltage drive model "RQ1C075UN". The Schottky diode used for the rectifier was "MBRS340".

The frequency used for the measurement and the position of the coil were the same as in the previous section. Fig. 14 shows the results of load voltage and efficiency measurement for each coupling coefficient. The transmission efficiency of the circuit of Fig. 13 is obtained by equation 3 .

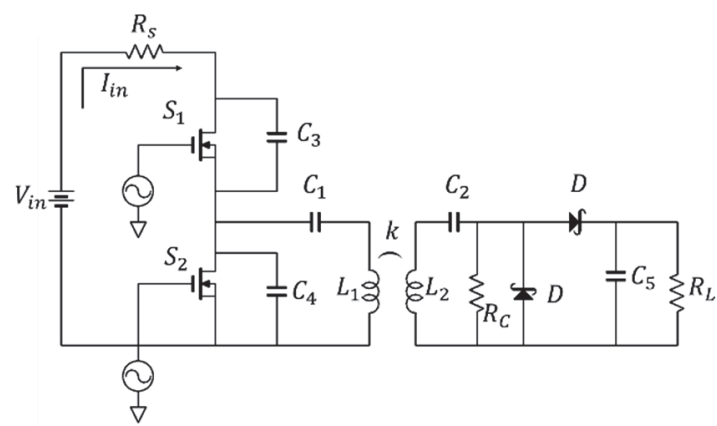

Fig. 13 Power transmission circuit using Class DE inverter.

Table 3 Experimental Circuit Components and Value.

\begin{tabular}{|c|c|c|}
\hline Components & Part & Value \\
\hline Power Supply Voltage & $V_{i n}$ & $5.0(\mathrm{~V})$ \\
\hline $\begin{array}{c}\text { Inductance of Transmitter } \\
\text { Coil }\end{array}$ & $L_{1}$ & $13.6(\mu \mathrm{H})$ \\
\hline Inductance of Receiver Coil & $L_{2}$ & $66.0(\mu \mathrm{H})$ \\
\hline Load Resistance & $R_{L}$ & $39.6(\Omega)$ \\
\hline $\begin{array}{c}\text { Transmitter side resonance } \\
\text { capacitor }\end{array}$ & $C_{l}$ & $0.18(\mu \mathrm{F})$ \\
\hline $\begin{array}{c}\text { Receiver side resonance } \\
\text { capacitor }\end{array}$ & $C_{2}$ & $0.04(\mu \mathrm{F})$ \\
\hline $\begin{array}{c}\text { Shunt capacitor } \\
\text { Smoothing capacitor }\end{array}$ & $C_{3}$ & $0.033(\mu \mathrm{F})$ \\
\cline { 2 - 3 } & $C_{4}$ & $0.032(\mu \mathrm{F})$ \\
\hline $\begin{array}{c}\text { Charge release resistance } \\
\text { Resistance for Current } \\
\text { Detection }\end{array}$ & $R_{C}$ & $1.0(\mu \mathrm{F})$ \\
\hline$R_{S}$ & $0.5(\mathrm{k} \Omega)$ \\
\hline
\end{tabular}




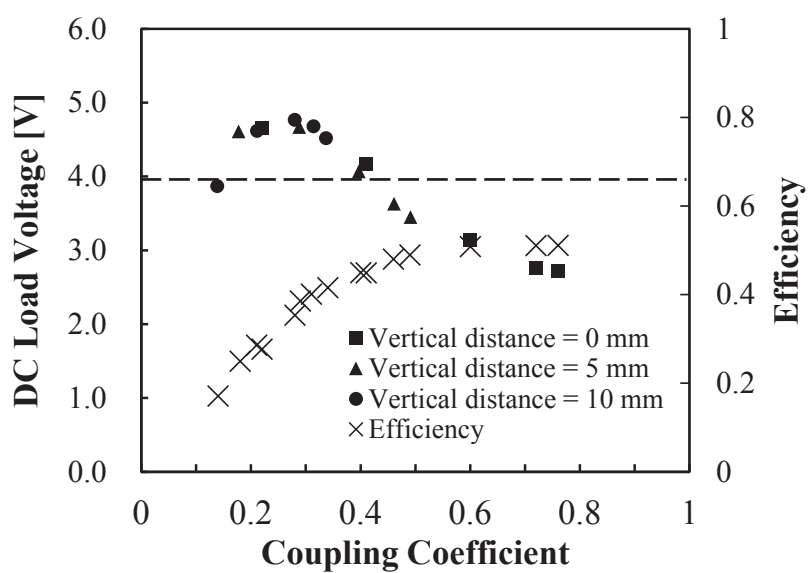

Fig. 14 Load voltage and efficiency at each coupling coefficient.

$$
\eta^{\prime}=\frac{\frac{V_{R L}{ }^{2}}{R_{L}}}{V_{i n} I_{i n}}
$$

From Fig. 14, it was 9 points that exceeded the reference voltage of $4.0 \mathrm{~V}$ out of the measured 15 points. The practical coupling coefficient range exceeding the reference voltage was 0.18 to 0.41 . The load voltage decreased, when the coupling coefficient exceeded 0.41. The maximum load voltage of $4.77 \mathrm{~V}$ was obtained when the coupling coefficient was 0.28 . Among the measuring points exceeding the reference voltage, the obtained maximum efficiency was $45 \%$ when the coupling coefficient was 0.41 .

\section{Conclusions and Future Works}

The manufactured methods and the electric characteristics of coils made by using the sewing techniques were described. The maximum coupling coefficient was 0.75 . The maximum efficiency of $75.0 \%$ was obtained when the coupling coefficient was the highest. In the measurement range, the efficiency was increased as the coupling coefficient approached one. The maximum load voltage was obtained $4.77 \mathrm{~V}$. The range of practical coupling coefficient exceeding the reference voltage of $4.0 \mathrm{~V}$ was 0.18 to 0.41 . The highest efficiency within that range was obtained at $45 \%$ when the coupling coefficient was 0.41 . The usefulness of the sewn coil as the transmission coil was confirmed in the coupling coefficient range 0.18 to 0.41 .

The future work is to increase transmission efficiency. Heat generation when charging the device can be reduced by improving efficiency. The efficiency strongly depends on the coupling coefficient of a coils and the quality factor. The quality factor of coils can be improved by reducing resistance or increasing the inductance. The loss resistance decreases by increasing the Litz wire diameter. The inductance increases as the number of turns is added. The loss resistance also increases when the number of turns of the coil is added. For that reason, it is important to select an appropriate Litz wire according to the desired size and winding number. Also, the transmission efficiency obtained with a practical coupling coefficient was $50 \%$ or less. In order to exceed the reference voltage with higher efficiency, the inverter should adopt the full bridge type.

\section{References}

[1] Gopi Krishna Durbhaka, "Adaptive wearable smart fabric based on body posture and temperature," Advances in Computing, Communication, \& Automation (ICACCA) (Fall), International Conference, pp.1-5, 2016.

[2] Saisai Wen, Hadi Heidari, Anastasios Vilouras, "A wearable fabric-based RFID skin temperature monitoring patch," IEEE SENSORS, pp1-3, 2016.

[3] Asimina Kiourti, Cedric Lee, John L. Volakis, "Fabrication of Textile Antennas and Circuits With 0.1 $\mathrm{mm}$ Precision," IEEE Antennas and Wireless Propagation Letters, vol. 15, pp.151-153, May. 2015.

[4] Dibin Zhu, Neil J.Grabham, Lindsay Clare, "Inductive power transfer in e-textile applications: Reducing the effects of coil misalignment," WPTC IEEE, pp1-4, 2015.

[5] Jimei Ma, Qingxin Yang, Haiyan Chen, "Transcutaneous Energy and Information Transmission System With Optimized Transformer Parameters for the Artificial Heart," IEEE Transaction on Power Electro., vol. 30, No. 11, pp.6211-6236, Nov. 2015.

[6] Ryohei Suwahara et al. "Production of the Coils by Use of Sewing Technologies for an Electromagnetic Induction Power Transmission," Journal of JSAEM, vol. 25, No. 2, pp100-105, 2017.

[7] Neil J. Grabham, Yi Li, Lindsay R. Clare, Bernard H. Stark, and Stephen P. Beeby, "Fabrication Techniques for Manufacturing Flexible Coils on Textiles for Inductive Power Transfer," IEEE SENSORS JOURNAL, Vol. 18, NO. 6, Mar. 15, pp2599-2606, 2018.

[8] Gerard J. Tortora, Bryan H. Derrickson (2008) Principles of Anatomy and Physiology, 12th Edition 12th Edition: John Wiley \& Sons

[9] Q. Deng, J. Liu, D. Czarkowski, M. K. Kazimierczuk, M. Bojarski, H.Zhou, and W. Hu, "Frequency-dependent resistance of Litz-wire square solenoid coils and quality factor optimization for inductive powertransfer," IEEE Trans. Ind. Electron., vol. 63, no. 5, pp. 2825-2837, May 2016

[10] C. R. Sullivan: "Optimal choice for number of strands in a Litz-wire transformer winding," IEEE Trans. Power Electron., vol. 14, no. 2, pp. 283-291, Mar. 1999.

[11] X. Nan and C. R. Sullivan, "An equivalent complex permeability model for Litz-wire windings," IEEE Trans. Ind. Appl., vol. 45, no. 2, pp. 854-860, Mar. 2009.

[12] WIRELESS POWER CONSORTIUM The Qi Wireless Power Transfer System Power Class 0 Specification Part 4: Reference Designs Version 1.2.2 April (2016), pp.5359.

[13] Marian K. Kazimierczuk, Dariusz Czarkowski, (2011) RSONANT POWER CONVERTERS, pp. 382-403 : John Wiley \& Sons. 